\title{
Disease-associated variants in different categories of disease located in distinct regulatory elements
}

Meng Ma ${ }^{1,4+}$, Ying $\mathrm{Ru}^{1,3+}{ }^{1}$, Ling-Shiang Chuang ${ }^{1}$, Nai-Yun Hsu', Li-Song Shi ${ }^{1}$, Jörg Hakenberg ${ }^{1}$, Wei-Yi Cheng ${ }^{1}$, Andrew Uzilov', Wei Ding ${ }^{1}$, Benjamin S Glicksberg ${ }^{1,2}$, Rong Chen ${ }^{1 *}$

From Varl-SIG 2014: Identification and annotation of genetic variants in the context of structure, function and disease

Boston, MA, USA. 12 July 2014

\begin{abstract}
Background: The invention of high throughput sequencing technologies has led to the discoveries of hundreds of thousands of genetic variants associated with thousands of human diseases. Many of these genetic variants are located outside the protein coding regions, and as such, it is challenging to interpret the function of these genetic variants by traditional genetic approaches. Recent genome-wide functional genomics studies, such as FANTOM5 and ENCODE have uncovered a large number of regulatory elements across hundreds of different tissues or cell lines in the human genome. These findings provide an opportunity to study the interaction between regulatory elements and disease-associated genetic variants. Identifying these diseased-related regulatory elements will shed light on understanding the mechanisms of how these variants regulate gene expression and ultimately result in disease formation and progression.
\end{abstract}

Results: In this study, we curated and categorized 27,558 Mendelian disease variants, 20,964 complex disease variants, 5,809 cancer predisposing germline variants, and 43,364 recurrent cancer somatic mutations. Compared against nine different types of regulatory regions from FANTOM5 and ENCODE projects, we found that different types of disease variants show distinctive propensity for particular regulatory elements. Mendelian disease variants and recurrent cancer somatic mutations are 22-fold and 10- fold significantly enriched in promoter regions respectively ( $q<0.001)$, compared with allele-frequency-matched genomic background. Separate from these two categories, cancer predisposing germline variants are 27 -fold enriched in histone modification regions $(\mathrm{q}<0.001)$, 10 -fold enriched in chromatin physical interaction regions $(q<0.001)$, and 6 -fold enriched in transcription promoters $(q<0.001)$. Furthermore, Mendelian disease variants and recurrent cancer somatic mutations share very similar distribution across types of functional effects.

We further found that regulatory regions are located within over 50\% coding exon regions. Transcription promoters, methylation regions, and transcription insulators have the highest density of disease variants, with 472 , 239, and 72 disease variants per one million base pairs, respectively.

Conclusions: Disease-associated variants in different disease categories are preferentially located in particular regulatory elements. These results will be useful for an overall understanding about the differences among the pathogenic mechanisms of various disease-associated variants.

\footnotetext{
* Correspondence: rong.chen@mssm.edu

+ Contributed equally

'Department of Genetics and Genomic Sciences, Icahn School of Medicine

at Mount Sinai, New York, NY, 10029, USA

Full list of author information is available at the end of the article
}

(c) 2015 Ma et al.; licensee BioMed Central Ltd. This is an Open Access article distributed under the terms of the Creative Commons 


\section{Background}

Along with the wide application of high throughput technologies, hundreds of millions genetic variants have been identified with a dramatic growth of dbSNP occurring after 2007 [1]. From these resources/studies, it was found that $\sim 97 \%$ of all identified variants are noncoding variants, consistent with the notion that $98 \%$ of human genome sequences are noncoding [2]. The studies that have resulted from the ENCODE project show that over $80 \%$ of human genome are functional [3], participating in at least one biochemical RNA- or chromatinassociated event in at least one cell type. Any variant that is located within a functional genomic region potentially has the ability to cause a dysregulation on gene expression through modifying regulatory elements, possibly resulting in diseases pathogenesis $[4,5]$. A lot of well-annotated disease-variants have been collected in the Human Gene Mutation Database (HGMD) [6]; these variants are organized into three groups of significant functional disease SNPs, namely coding SNPs (cSNPs), splicing SNPs (sSNPs) and regulatory SNPs (rSNPs), which account for $\sim 86 \%, \sim 10 \%$ and $\sim 3 \%$ of variants in HGMD respectively [6-9]. There is plenty of information about coding variants but limited knowledge about noncoding variants. In recent years, genome-wide association studies (GWAS) [10] identified over ten thousand variants associated with various diseases/traits, $~ 90 \%$ of which localize outside of known protein-coding regions. This phenomenon highlights the substantial gap between the plethora of disease- or trait-associated noncoding variants and our understanding of how most of these variants contribute to diseases/traits. (Figure S1).

Gene expression is a tightly regulated process, involving various regulatory elements including promoters, enhancers, insulators, and silencers. Moreover, the chemical modifications (i.e. methylation and acetylation) on histone proteins present in chromatin has been shown to change the accessibility of the chromatin for transcription to occur and thusly influence gene expression [11,12]. Some projects, such as ENCODE [3] and FANTOM5 [13,14], adopted various experimental technologies including ChIP- seq [15], DNase-seq [16], ChIA-PET [17], and CAGE [18-21], and identified a lot of various regulatory regions throughout the human genome across hundreds of tissues and cell types [22]. These various experiments validated regulatory regions datum provide an opportunity to investigate the underlying pathogenic mechanism of disease-associated variants.

A possible mechanism underlying the pathogenesis of disease-associated variants is the disruption of the binding of transcription factors, local chromatin structure, and/or co-factors recruitment, ultimately altering the expression of the target genes. Some published studies support such a hypothesis through analyzing the distribution of regulatory complex disease variants by GWAS [3,23-30]. In the current study, we focus on the dissimilarity of underlying pathogenic regulatory mechanisms of disease-associated variants in different disease categories, including Mendelian diseases, complex diseases, cancer predisposing germline variants, and recurrent cancer somatic mutations.

\section{Results and discussion}

Distinct densities of disease-associated variants within different types of regulatory regions

Curation of disease-associated variants and regulatory regions

We curated disease-associated variants for Mendelian diseases, germline cancers, somatic cancers, and complex diseases (see details in supplementary materials, Additional File 1). Disease-associated variants are summarized in Table 1. There were 27,558 Mendelian disease variants collected from OMIM [31] and ClinVar [32], residing within 2,229 genes and causing 5,317 diseases/phenotypes. VarDi is a database of disease-associated variants built through a combination of Hadoop-based text mining tools and manual curation [33]. We collected 20,964 complex disease variants from VarDi and NHGRI GWAS Catalog [10], located within 2,615 genes and associated with 1,243 diseases/traits. Compared with 5,809 cancer predisposing germline variants from HGMD professional database [6], 43,364 recurrent cancer somatic mutations were extracted from COSMIC [34]. Cancer predisposing germline variants were located across 294 genes, while recurrent cancer somatic mutations were distributed throughout 14,649 genes.

A lot of regulatory regions, including transcription promoters, enhancers and insulators, DNA methylation regions, histone modification regions, chromatin physical interaction regions, DNA binding sites of protein factors by ChIP-seq, and open chromatin regions by DNase-seq and FAIRE-seq, were identified by the FANTOM5 and ENCODE projects, which are summarized in Table 2. Transcription promoter, enhancer, and insulator regions account for $0.12 \%, 0.38 \%$ and $3.52 \%$ of the human genome respectively. Roughly $0.6 \%$ of the human genome is DNA methylation regions, usually overlapping with transcription promoter regions. Histone modification regions occupy over $87 \%$ of the human genome, revealing the ubiquity of epigenetic marked regions. About $40 \%$ of the human genome involves chromatin physical interaction zones, hinting an abundant, longrange regulation during gene expression process. Similar percentages of human genome, $11.76 \%, 11.97 \%$ and $13.87 \%$, comprise DNA binding sites of protein by ChIP-seq and open chromatin regions by DNase-seq and FAIRE-seq, respectively. 
Table 1. Summary of disease variants

\begin{tabular}{lllll}
\hline Variants & Data sources & \#Variants & \#Genes & \#Diseases/Phenotypes/Traits \\
\hline Mendelian disease variants & OMIM, ClinVar & 27,558 & 2,229 & 5,317 \\
Complex disease variants & GWAS catalog, VarDi & 20,964 & 2,615 & 1,243 \\
Cancer predisposing germline variants & HGMD professional & 5,809 & 294 & 260 \\
Recurrent cancer somatic mutations & COSMIC & 43,364 & 14,649 & 296 \\
\hline
\end{tabular}

\section{Regulatory regions are widely located within coding and noncoding regions}

We extracted seven types of human genomic regions: coding exons, 5'-UTR, 3'-UTR, introns, upstream and downstream 2000bp of genes, and intergenic region; and then counted the regulatory regions overlapping with each type of the genomic regions (Table 3, S1). Regulatory regions are widely located within different human genomic regions, and each type of human genomic regions also can contain various regulatory regions. Transcription promoters can occur within each type of genomic region. $\sim 45 \%$ of promoters can be within intergenic regions, hinting a lot of potential protein or RNA genes unknown in the intergenic regions. Intronic promoters account for $\sim 24 \%$, consistent with the study that lots of latent noncoding RNA genes within introns [35]; Intronic promoters are associated with various disorders, including cancer [36]. Dr. Ingolia found pervasive translation outside of annotated protein-coding genes through ribosome profiling analysis, implying that a lot of transcription promoters are located outside of protein-coding genes [37]. Transcription enhancers and insulators mainly locate within intergenic regions, introns, upstream and downstream of genes. Over $15 \%$ of methylation regions occurs within coding exons, 5'-UTR and upstream of genes. The majority of regulatory regions are located within noncoding regions including introns and intergenic regions, while coding regions also contain various regulatory regions. $54.99 \%$ of coding exons are overlapped with regulatory regions, implying the regulatory role of coding exons on gene expression. $\sim 15 \%$ of coding exon regions can be DNA binding sites of proteins, which is in agreement with the study that genetic code specifying amino acids and regulatory code specifying transcription factor recognition sequences has been proven to exist simultaneously within human protein coding regions [38]. Regulatory activity on gene expression can occur within any type of human genomic regions.

Illumina SureSelect TruSeq and Nimblegen SeqCap EZ are two popular exome DNA sequencing technologies which can be used to identify Mendelian disease variants, cancer predisposing germline mutations and cancer somatic mutations. The target regions of these two exome DNA sequencing platforms can be located within various human genomic regions (Table S2, S3). Moreover, these target regions also are overlapped with various regulatory regions (Table S4, S5), suggesting any disease variants identified by such exome DNA sequencing platform can likely be located within any type of regulatory regions.

Table 2. Summary of regulatory regions from FANTOM5 and ENCODE

\begin{tabular}{|c|c|c|c|c|c|}
\hline Regulatory Regions & Source & Technique & Institute & Length(bp) & $\begin{array}{l}\text { Percent of } \\
\text { human } \\
\text { genome (\%) }\end{array}$ \\
\hline Transcription promoter & FANTOM5 & CAGE & RIKEN & $3,833,500$ & 0.12 \\
\hline Transcription enhancer & FANTOM5 & CAGE & RIKEN & $12,385,403$ & 0.38 \\
\hline Transcription insulator & ENCODE & ChIP-seq & $\begin{array}{l}\text { HudsonAlpha Institute for Biotechnology, Yale University, Harvard } \\
\text { University }\end{array}$ & $81,713,060$ & 3.52 \\
\hline Methylation region & ENCODE & $\begin{array}{l}\text { Methylation } \\
450\end{array}$ & HudsonAlpha Institute for Biotechnology & $19,517,834$ & 0.60 \\
\hline $\begin{array}{l}\text { Histone modification } \\
\text { region }\end{array}$ & ENCODE & ChlP-seq & $\begin{array}{l}\text { Broad institute, Massachusetts General Hospital, Harvard Medical } \\
\text { School }\end{array}$ & $2,816,878,674$ & \\
\hline $\begin{array}{l}\text { Chromatin physical } \\
\text { interaction regions }\end{array}$ & ENCODE & CHIA-PET & Genome Institute of Singapore, Stanford University & $1,288,430,643$ & 39.83 \\
\hline $\begin{array}{l}\text { DNA binding sites of } \\
\text { protein }\end{array}$ & ENCODE & ChIP-seq & $\begin{array}{l}\text { HudsonAlpha Institute for Biotechnology, Yale University, Harvard } \\
\text { University }\end{array}$ & $380,355,257$ & 11.76 \\
\hline $\begin{array}{l}\text { Open chromatin } \\
\text { regions (DNase I } \\
\text { hypersensitive sites) }\end{array}$ & ENCODE & DNase-seq & Washington University, Duke University & $387,138,495$ & 11.97 \\
\hline $\begin{array}{l}\text { Open chromatin } \\
\text { regions by FAIRE-seq }\end{array}$ & ENCODE & FAIRE-seq & $\begin{array}{l}\text { Duke University, University of North Carolina at Chapel Hill, } \\
\text { University of Texas at Austin, European Bioinformatics Institute, } \\
\text { University of Cambridge }\end{array}$ & $448,557,442$ & 13.87 \\
\hline
\end{tabular}


Table 3. Percentage of each types of regulatory regions overlapped with different human genomic regions

\begin{tabular}{|c|c|c|c|c|c|c|c|}
\hline & $\begin{array}{l}\text { Overlapping } \\
\text { Coding Exon } \\
\text { (bp) }\end{array}$ & $\begin{array}{l}\text { Overlapping } \\
\text { Upstream (bp) }\end{array}$ & $\begin{array}{l}\text { Overlapping } \\
\text { 3'-UTR (bp) }\end{array}$ & $\begin{array}{l}\text { Overlapping } \\
\text { 5'-UTR (bp) }\end{array}$ & $\begin{array}{l}\text { Overlapping } \\
\text { Introns (bp) }\end{array}$ & $\begin{array}{l}\text { Overlapping } \\
\text { Downstream } \\
\text { (bp) }\end{array}$ & $\begin{array}{l}\text { Overlapping } \\
\text { Intergenic Regions } \\
\text { (bp) }\end{array}$ \\
\hline Transcription & 318,864 & $1,033,885$ & 235,904 & 793,623 & 948,521 & 202,588 & $1,747,482$ \\
\hline promoter & $(8.32 \%)$ & (26.97\%) & (6.15\%) & (20.7\%) & $(24.74 \%)$ & $(5.23 \%)$ & (45.58\%) \\
\hline Transcription & 275 & 443,003 & 12,826 & 8,301 & $4,351,818$ & 326,034 & $7,628,892$ \\
\hline enhancer & $(0.002 \%)$ & (3.58\%) & $(0.1 \%)$ & $(0.07 \%)$ & (35.14\%) & $(2.63 \%)$ & $(61.6 \%)$ \\
\hline Transcription & $1,678,883$ & $6,568,355$ & $1,642,952$ & $2,007,230$ & $25,080,619$ & $3,312,161$ & $49,525,266$ \\
\hline insulator & $(2 \%)$ & (8.04\%) & $(2 \%)$ & $(2.45 \%)$ & (30.7\%) & $(4.05 \%)$ & $(60.6 \%)$ \\
\hline Methylation & 961,891 & $3,066,144$ & 790,094 & 850,738 & $5,552,194$ & $1,039,107$ & $10,745,614$ \\
\hline region & $(4.93 \%)$ & $(15.71 \%)$ & $(4.04 \%)$ & $(4.36 \%)$ & $(28.45 \%)$ & $(5.32 \%)$ & $(55 \%)$ \\
\hline Histone & $19,836,913$ & $65,289,808$ & $27,693,911$ & $13,261,068$ & $901,286,244$ & $69,178,945$ & $1,820,842,829$ \\
\hline modification region & $(0.7 \%)$ & $(2.32 \%)$ & $(1 \%)$ & $(0.47 \%)$ & $(32 \%)$ & $(2.46 \%)$ & $(64.64 \%)$ \\
\hline Chromatin & $14,486,785$ & $45,832,380$ & $20,066,412$ & $9,708,604$ & $464,537,271$ & $46,072,176$ & $761,585,471$ \\
\hline $\begin{array}{l}\text { physical interaction } \\
\text { regions }\end{array}$ & $(1.12 \%)$ & $(3.56 \%)$ & $(1.6 \%)$ & $(0.75 \%)$ & (36.05\%) & $(3.58 \%)$ & $(59.1 \%)$ \\
\hline DNA binding & $5,252,509$ & $22,329,204$ & $6,553,411$ & $5,567,759$ & $130,492,231$ & $14,945,226$ & $224,621,692$ \\
\hline sites of protein & $(1.38 \%)$ & $(5.87 \%)$ & $(1.7 \%)$ & (1.46\%) & (34.3\%) & (3.93\%) & (59.06\%) \\
\hline Open & $6,309,327$ & $22,046,177$ & $6,966,217$ & $5,626,301$ & $131,734,716$ & $15,003,875$ & $229,306,915$ \\
\hline $\begin{array}{l}\text { chromatin regions } \\
\text { (DNase }\end{array}$ & $(1.63 \%)$ & $(5.69 \%)$ & $(1.8 \%)$ & $(1.45 \%)$ & (34.02\%) & $(3.88 \%)$ & $(59.23 \%)$ \\
\hline \multicolumn{8}{|l|}{ I hypersensitive sites) } \\
\hline Open & $4,896,207$ & $18,119,824$ & $6,098,267$ & $4,612,397$ & $137,079,859$ & $12,725,401$ & $290,428,922$ \\
\hline $\begin{array}{l}\text { chromatin regions by } \\
\text { FAIRE-seg }\end{array}$ & $(1.09 \%)$ & $(4.03 \%)$ & $(1.4 \%)$ & $(1.02 \%)$ & $(30.56 \%)$ & $(2.84 \%)$ & $(64.75 \%)$ \\
\hline
\end{tabular}

\section{Highest density of disease-associated variants within transcription promoter}

We counted the number of disease-associated variants within each type of regulatory regions, and calculated the average number of disease variants per one million base pairs of regulatory regions (DVPM) (Table 4). The highest density of disease-associated variants was in transcription promoter (472 DVPM), which is reasonable considering the importance of transcription promoters in initiating gene expression. Methylated regions had a DVPM of 239, which usually overlap with transcription promoter regions. Transcription insulator had the third highest density of 72 DVPM, while transcription enhancer region had the lowest DVPM of 18. Transcription insulator regions are tightly associated with the 3D structure of DNA, mediated by CTCF protein. Accordingly, variants in insulator regions can result in changes of the 3D structure of DNA [39]. The density of disease variants in other types regulatory regions range from 33 to 68 . The disease-associated variants have quite different densities in various regulatory regions.

\section{Similar pattern of functional effects between Mendelian} disease variants and recurrent cancer somatic mutations We applied Ensebml Variants Effect Predictor [40] to annotate the functional effects of disease variants in four disease categories (Figure 1A-D). Functional effects of variants can be classified into 34 consequences in Sequence Ontology [41], which were ranked in the order of severity (more severe to less severe) by Ensembl analysis group [40,42] (Figure 1E, Table S6). Mendelian disease variants and recurrent cancer somatic mutations share the same top functional effects: missense_variant ( $24 \%$ vs $28 \%)$, downstream_gene_variant (18\% vs $13 \%)$, upstream_gene_variant (11\% vs $8 \%)$, nc_transcript_variant ( $10 \%$ vs $11 \%)$, non_coding_exon_variant ( $8 \%$ vs $8 \%)$, intron_variant (6\% vs 7\%), NMD_transcript_variant ( $4 \%$ vs $5 \%$ ) and stop_gained (5\% vs $3 \%$ ). Mendelian disease variants and recurrent cancer somatic mutations show similar pattern of functional effects.

The majority of complex disease variants are noncoding variants. Intron_variant (46\%), upstream_gene_variant (10\%), downstream_gene_variant (10\%) and intergenic_variant (8\%) sum up to $\sim 75 \%$ of the overall complex disease variants. Considering that complex disease variants identified via GWAS are not necessarily the causal variants, and functional annotation of the GWAS SNPs may not reflect the nature of complex disease causal variants, we further recompiled the annotation on those complex disease variants that were replicated in at least two different ethnicities, and more likely to be causal than just markers. We produced a similar annotation result for complex disease causal variants (Figure S2). Intron_variant (39\%), upstream_gene_variant (19\%), downstream_gene_variant 
Table 4. Summary of disease variants residing within regulatory regions in seven types of human genomic regions

\begin{tabular}{|c|c|c|c|c|c|c|c|c|c|}
\hline & $\begin{array}{l}\text { \#Disease } \\
\text { Variants } \\
\text { within } \\
\text { upstream }\end{array}$ & $\begin{array}{l}\text { \#Disease } \\
\text { Variants } \\
\text { within } \\
\text { 5'UTR }\end{array}$ & $\begin{array}{l}\text { \#Disease } \\
\text { Variants } \\
\text { within coding } \\
\text { exons }\end{array}$ & $\begin{array}{l}\text { \#Disease } \\
\text { Variants } \\
\text { within } \\
\text { introns }\end{array}$ & $\begin{array}{l}\text { \#Disease } \\
\text { Variants } \\
\text { within } \\
\text { 3'UTR }\end{array}$ & $\begin{array}{l}\text { \#Disease } \\
\text { Variants } \\
\text { within } \\
\text { downstrea m }\end{array}$ & $\begin{array}{l}\text { \#Disease } \\
\text { Variants within } \\
\text { intergenic } \\
\text { region }\end{array}$ & $\begin{array}{l}\text { Total } \\
\text { (unique } \\
\text { variants) }\end{array}$ & $\overline{\text { DVPM }}$ \\
\hline $\begin{array}{l}\text { Transcription } \\
\text { promoter }\end{array}$ & 666 & 589 & 1,675 & 480 & 555 & 691 & 7 & 1,812 & 472 \\
\hline Methylation region & 1,378 & 1,062 & 4,113 & 1,440 & 1,196 & 1,317 & 48 & 4,671 & 239 \\
\hline $\begin{array}{l}\text { Transcription } \\
\text { insulator }\end{array}$ & 1,876 & 1,472 & 4,637 & 1,976 & 1,393 & 1,790 & 279 & 5,886 & 72 \\
\hline $\begin{array}{l}\text { Open chromatin } \\
\text { regions (DNase I } \\
\text { hypersensitiv e } \\
\text { sites) }\end{array}$ & 6,661 & 5,776 & 20,306 & 9,580 & 7,334 & 7,336 & 1214 & 26,188 & 68 \\
\hline $\begin{array}{l}\text { DNA binding sites } \\
\text { of protein }\end{array}$ & 6,360 & 5,122 & 17,211 & 9,043 & 6,181 & 7,016 & 1143 & 22,915 & 60 \\
\hline $\begin{array}{l}\text { Chromatin physical } \\
\text { interaction regions }\end{array}$ & 13,775 & 13,040 & 49,007 & 26,125 & 19,005 & 17,952 & 3447 & 65,309 & 51 \\
\hline $\begin{array}{l}\text { Open chromatin } \\
\text { regions by FAIRE- } \\
\text { seq }\end{array}$ & 5,612 & 5,013 & 16,922 & 8,779 & 6,434 & 6,261 & 1203 & 22,470 & 50 \\
\hline $\begin{array}{l}\text { Histone } \\
\text { modification region }\end{array}$ & 16,862 & 16,783 & 69,303 & 35,889 & 24,566 & 22,090 & 6196 & 93,212 & 33 \\
\hline $\begin{array}{l}\text { Transcription } \\
\text { enhancer }\end{array}$ & 30 & 1 & 0 & 129 & 1 & 12 & 71 & 219 & 18 \\
\hline
\end{tabular}

DVPM: the average number of disease variants per one million base pairs regulatory regions.

(19\%), and intergenic_variant (4\%), sum up to $~ 80 \%$ of the overall complex disease causal variants, supporting complex disease causal variants mainly are located within noncoding region.

More deleterious functional effects are found for Mendelian disease variants, cancer predisposing germline variants, and recurrent cancer somatic mutations compared to complex disease variants. Deleterious functional effects, such as stop_gained and frameshift_variant make up a substantial part of recurrent cancer somatic mutations, cancer predisposing germline variants and Mendelian disease variants. We generated a histogram of the functional effects of the four types of disease variants (Figure 1E). Roughly 5\% of cancer predisposing germline variants change splice sites, suggesting abnormal splicing isoforms caused by variants might lead to cancer formation. Stop_gained variants may result in a prematurely ended protein product, which is notable among the consequences of Mendelian disease variants, cancer predisposing germline variants and recurrent cancer somatic mutations. The top eleven serious consequences, specifically transcript_ablation, splice_donor_variant, splice_acceptor_variant, stop_gained, frameshift_variant, stop_lost, initiator_codon_variant, transcript_amplication, inframe_insertion, inframe_deletion and missense_variant (Figure 1E, Table S4), account for 32.39\%, 31.95\%, 30.55\% and $1.8 \%$ in cancer predisposing germline variants, recurrent cancer somatic mutations, Mendelian disease variants, and complex disease variants respectively. The majority of complex disease variants were annotated by the bottom fifteen consequences categories, suggesting milder functional effect of complex disease variants compared to other three types of disease variants. Accordingly, cancer predisposing germline variants, recurrent cancer somatic mutations, and Mendelian disease variants tend to cause more serious consequences compared to complex disease variants.

A limitation of this analysis is that the SNPs, which are linkage disequilibrium with complex disease variants, were not considered for the functional effect annotation analysis. Even so, we still accept that the complex disease associated variants can reflect the main properties of the disease-associated linkage disequilibrium genomic regions where the complex disease causal variants may locate. Therefore, this functional effect annotation analysis here can be helpful to understand the dissimilarity among the functional effects of the four types of diseaseassociated variants.

\section{Positive correlation between functionality of disease variants and evolutionary constraints on the disease variants}

A series of bioinformatics tools have been developed to predict whether variants are functional or deleterious. We applied GWAVA [43], Mutation Assessor [44], CADD [45], and GERP $[46,47]$ to score and measure the functionality of the four types of disease variants.

The functionalities of cancer predisposing germline variants, Mendelian disease variants, and recurrent cancer somatic mutations are greater than that of complex 


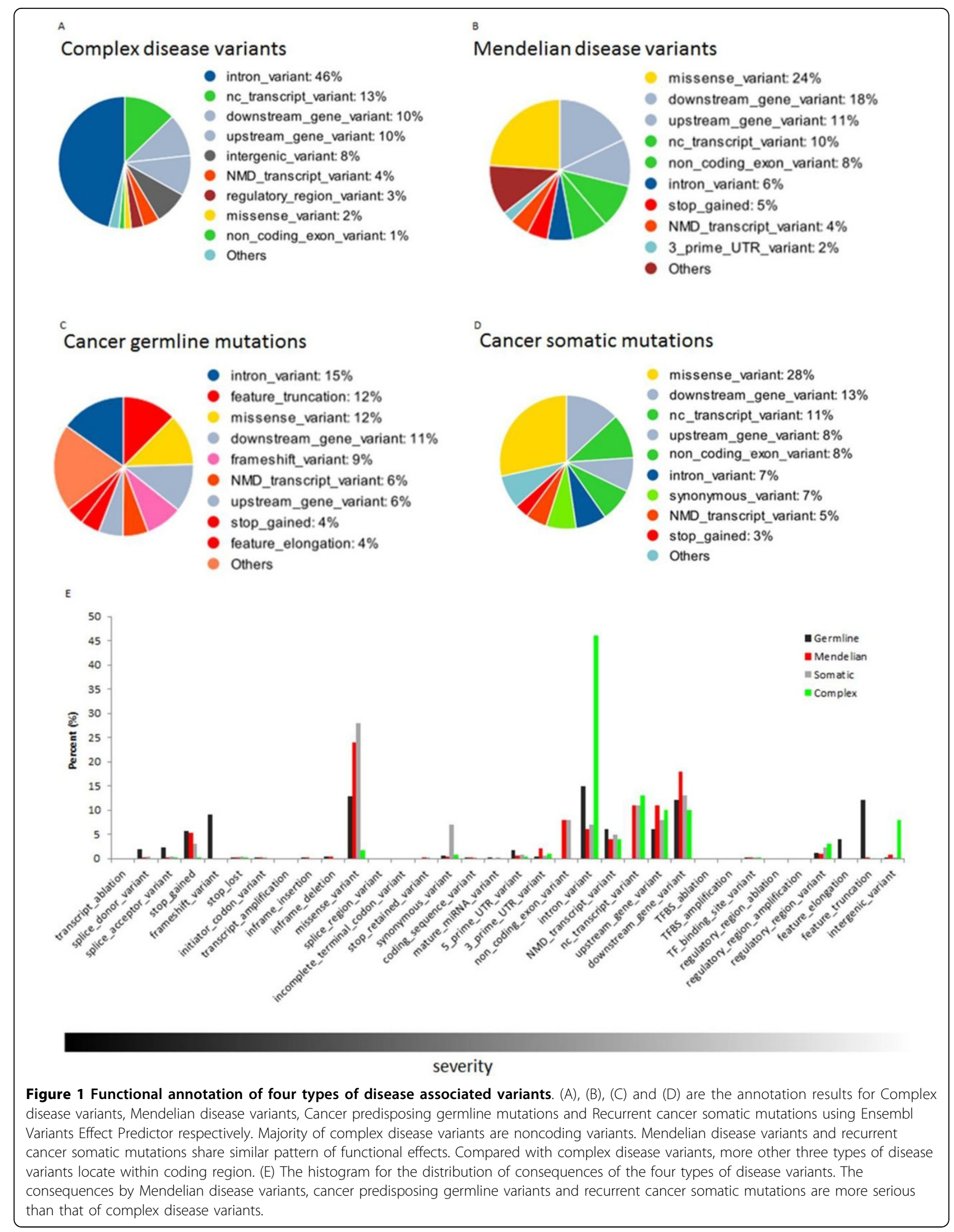


disease variants. GWAVA aims to predict functionality of noncoding variants. There are three kinds GWAVA scores: Region score, TSS score, and Unmatched score. A high GWAVA score means more active functionality with respect to a low GWAVA score. On the whole, the functionality of noncoding disease variants degrades in the order of germline cancer, Mendelian disease, somatic cancer, and complex disease (Figure 2A,B,C). Mutation Assessor predicts the functional impact of coding variants on the protein level. Usually functional coding variants have a higher Mutation Assessor score than non-functional coding variants. Mutation Assessor score threshold of 1.9 was used to discriminate disease-associated variants with medium or high functionality [44]. Within all the coding disease variants, we found that $55.31 \%$ of Mendelian disease variants, $44.65 \%$ of recurrent cancer somatic mutations, $36.2 \%$ of cancer predisposing germline variants, and $16.69 \%$ of complex disease variants are with medium or high functionality (Figure 2D). In general, the associated coding variants for germline cancer, Mendelian disease and somatic cancer are more functional than the associated coding variants for complex disease. CADD integrates multiple annotations to score the deleteriousness of coding or noncoding variants in the human genome. A high CADD score typically suggests more sever deleteriousness compared to a low CADD score. Some recurrent cancer somatic mutations have a very high CADD score, implying exceptional deleteriousness. By and large, however, Mendelian disease variants are the most deleterious. Complex disease variants in comparison are mild. Some recurrent cancer somatic mutations have a low, negative CADD score, and as such, are most likely neutral (Figure 2E). Overall, the deleteriousness of disease variants gradually increases in the order: complex disease variants, recurrent cancer somatic mutations, cancer predisposing germline variants, and Mendelian disease variants. The prediction score annotations for the four types of disease variants by GWAVA, Mutation Assessor, and CADD all suggest that cancer predisposing germline variants, Mendelian disease variants and recurrent cancer somatic mutations are more functional than complex disease variants.

Functional disease-associated variant is prone to under the evolutionary constraint. GERP $[46,47]$ can produce position-specific estimates of evolutionary constraint. Negative GERP scores indicate that a site is most likely evolutionary neutral. Positive scores suggest that a site may be under evolutionary constraint. Positive scores scale with the level of constraint, such that the greater the score, the greater the level of evolutionary constraint on that site. We found that $82.41 \%$ of cancer predisposing germline variants, $86.06 \%$ of Mendelian disease variants, $70.22 \%$ of recurrent cancer somatic mutations have a positive GERP score, while $\sim 60 \%$ of complex disease variants have a negative GERP score (Figure 2F), indicating that variants in the former group are under evolutionary constraint, while the majority of complex disease variants are evolutionary neutrally. Moreover, GWAVA, Mutation Assessor and CADD annotations of the four types of disease variants all suggest that the functionality of cancer predisposing germline variants, Mendelian disease variants, and recurrent cancer somatic mutations is greater than that of complex disease variants. By and large, the GERP score of the disease variants gradually decrease in the order of Mendelian disease variants, cancer predisposing germline variants, recurrent cancer somatic mutations, and complex disease variants. Thus, the aforementioned observations rationally lead to the conclusion that the greater the functionality of the disease variant, the greater the level of evolutionary constraint.

\section{Disease-associated variants in different disease categories are located within particular regulatory regions}

There is a pressing need to understand the pathogenic mechanism of disease-associated variants along with the wide application of high throughput sequencing technologies. Disease-associated variants located within regulatory regions, which cause dysregulation of the gene expression process, and result in abnormal protein products, is an important and efficient pathogenic mechanism. Therefore, the disease variants should be enriched within regulatory regions when compared to a control human genome variant background. The human genome variant background was generated as control group by subtracting four types of disease-associated variant from all SNPs that appear in the dbSNP database. We applied an odds ratio to measure the enrichment of disease variants within regulatory regions. We then plotted the natural logarithm of the odds ratios of disease variants to the control genome variant background within various regulatory regions (Figure 3A), which were tested statistically using a Pearson chi-squared test (Table S7).

Overall, the enrichments of different types of disease variants within various regulatory regions are different from each other. Enrichment of Mendelian disease variants, recurrent cancer somatic mutations, and cancer predisposing germline variants within transcription promoter regions are 21 times ( $\log$ value 3.04), 10.57 times ( $\log$ value 2.36) and 6.1 times (log value 1.8) higher than that of the genome variant background respectively, in contrast to only 1.9 times (log value 0.64 ) for complex disease variants. This implies that transcription promoters might be an efficient mechanism for Mendelian disease and cancer (germline or somatic), but not for complex disease pathogenesis. Additionally, the enrichment profile of the four types of disease variants within methylation regions just like that within transcription promoters. Mendelian 


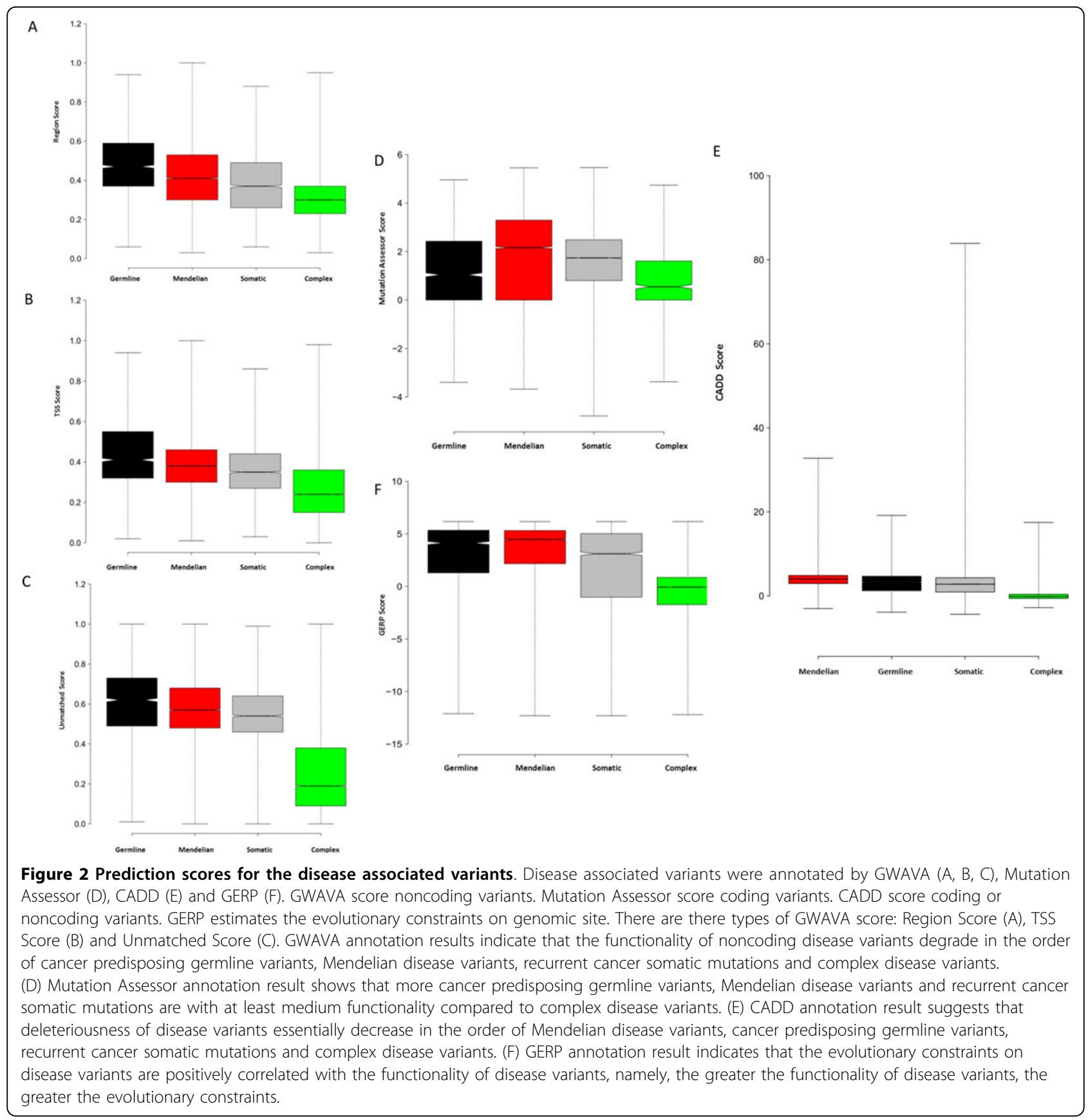

disease variants, recurrent cancer somatic mutations, and complex disease variants show higher enrichment within transcription insulator regions than cancer predisposing germline variants. Most disease variants are enriched within methylation and histone modification regions, suggesting a strong correlation between epigenetic marks and diseases, a pattern that some recent studies support [48-50]. In fact, cancer predisposing germline variants are over ten times more enriched within histone modification regions and chromatin physical interaction regions. There are no prominently enriched regulatory regions for complex disease variants, which present quite even enrichment distribution throughout all types of regulatory regions. Interestingly, complex disease variants show a positive enrichment within transcription enhancer, while other types of diseases variants have low negative enrichment, suggesting transcription enhancers might play an important role during complex disease development compared to other types of diseases. All four types of disease variants are enriched within DNA binding sites of protein by ChIP-seq, DNase I hypersensitive sites by DNase-seq, and open chromatin regions by FAIRE-seq. Disease- 


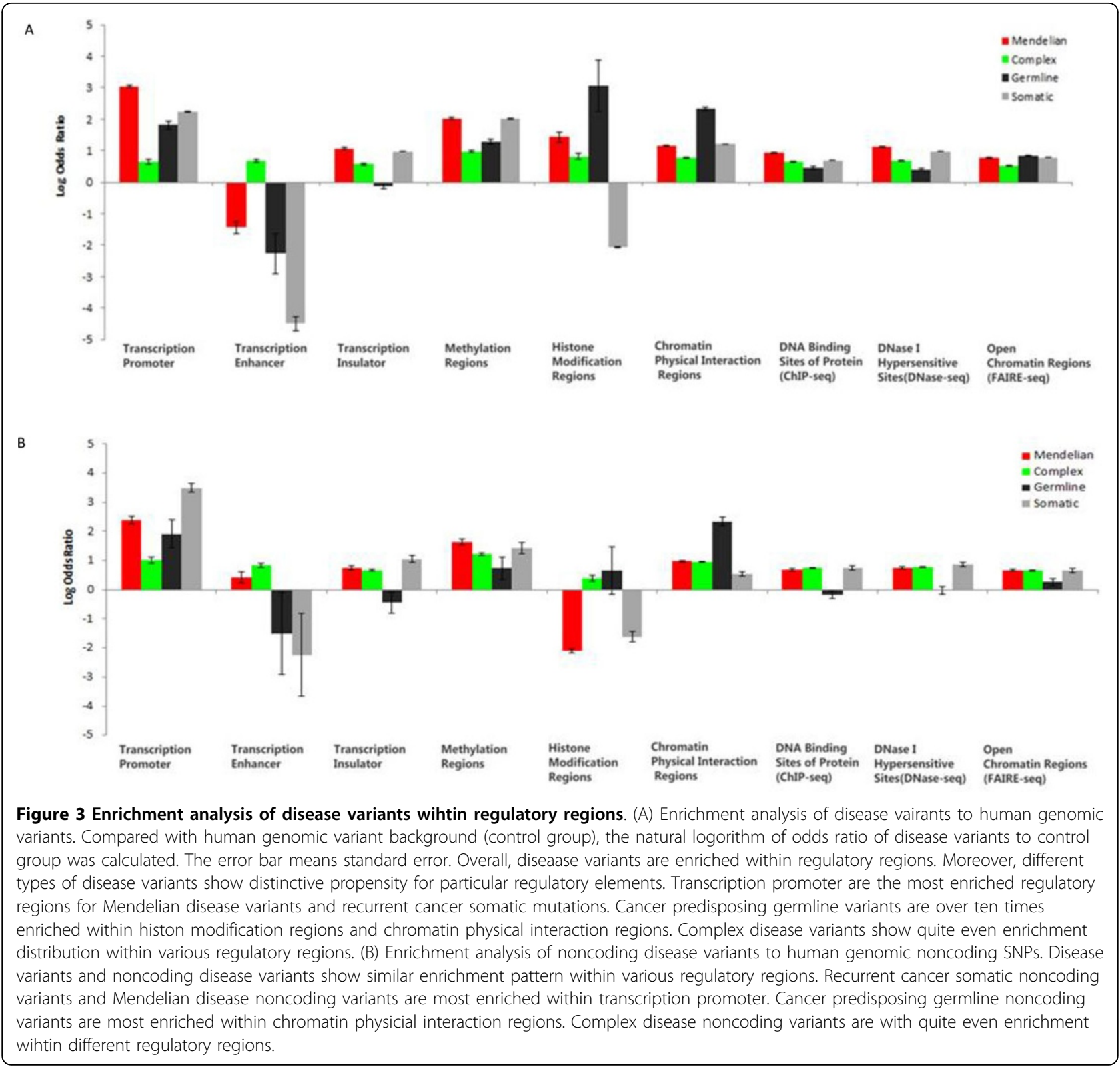

associated variants in different disease categories show dissimilar enrichment patterns within diverse regulatory elements, implying distinct priority of regulatory pathogenic mechanisms for different type of disease variants.

Considering that the majority of regulatory regions are located outside coding regions, and the distinct ratios of coding and noncoding disease variants in four types of disease categories may cause an acquisition bias on enrichment analysis, we further recalculated the enrichment analysis for only noncoding disease variants in four types of disease categories to eliminate the potential acquisition bias (Figure 3B, Table S8). By and large, the enrichment profile of noncoding disease variants is similar to that of all disease variants. Noncoding disease variants for Mendelian disease and cancer (germline or somatic) shows high enrichments within transcription promoter. Noncoding cancer germline variants are over ten times enriched within chromatin physical interaction regions. The highest enrichment within transcription enhancer is from complex disease variants. The outstanding enrichment difference between all disease variants and noncoding disease variants, occurs within histone modification regions, a dramatic decrease of enrichments, which conversely implies a tight association between histone modification epigenetic marks and disease variants that are located within coding regions. A recent study showed that histone modifications marks can be used to predict coding exon inclusion levels [51], which supports 
the idea that if the histone modification regions are altered by disease variants, then the change of target exons expression can be expected, potentially leading to disease formation. On the whole, noncoding disease variants and all disease variants show similar enrichment profiles within various regulatory regions.

Different types of disease-associated variants show distinctive propensity for particular regulatory elements. We generated specific control groups for each type of disease variants to identify particular regulatory regions where disease-associated variants are enriched according to the following steps. Firstly, we generated the distribution of the allele frequencies for each type of disease variants based on the third phase of the 1000 genome project [52]. Secondly, for each type of disease variants, we randomly selected 1000 equal size control groups which share the same allele frequency distribution of disease variants. Next, for each type of disease variants, we calculated the odds ratios of disease variants to 1000 equal size specific control groups and statistically calculated each odds ratio using Pearson chi-squared test under $\mathrm{p}$ value threshold 0.05 . Calculation of $q$ value was based on the $p$ values of 1000 enrichment analyses for each type of disease variant. The boxplot of the odds ratios for each type of disease variants are displayed in Figure 4. The Mendelian disease variants and recurrent cancer somatic mutations are most enriched in transcription promoter regions with median odds ratios of 22.1 and 10.87 respectively (Figure $4 \mathrm{~A}, \mathrm{C}$ ). Cancer predisposing germline variants have a median odds ratio of 26.5 in histone modification regions, 10.1 in chromatin physical interaction regions, and 6.46 in transcription promoter respectively (Figure 4B). Complex disease variants have a quite even enrichment distribution within the various regulatory regions (Figure 4D). We further repeated such analysis for only noncoding disease variants (Figure S3). Noncoding disease variants for Mendelian disease and germline cancer are most enriched within transcription promoter with median odds ratios of 8.96 and 18.82 respectively (Figure S3A, S3C). A dramatic drop in enrichment occurs from all cancer predisposing germline variants to noncoding cancer predisposing germline variants (Figure 4B, S3B). Noncoding cancer predisposing germline variants present median odds ratio 1.2 within histone modification regions against 26.5 of all cancer predisposing germline variants. Noncoding cancer predisposing germline variants still show relatively high enrichments within chromatin physical interaction regions and transcription promoters with a median odds ratio of 3.04 and 2.34, respectively. Noncoding complex disease variants still present quite even enrichment distribution (Figure S3D). The analysis result for the complex disease variants replicated in at least two different ethnicities, further confirms that no particular enriched regulatory region for complex disease variants (Figure S4). The enrichment analysis for all disease variants or noncoding disease variants based on allele-frequencymatched genomic background, indicate the distinct particular enriched regulatory regions for different types of disease variants.

The two types of enrichment analyses of disease variants, based on dbSNP control group and 1000 equal size specific control groups, both suggest that diseaseassociated variants in different disease categories preferentially locate within particular regulatory regions.

\section{Conclusions}

We curated 27,558 Mendelian disease variants, 20,964 complex disease variants, 5,809 cancer predisposing germline variants, and 43,364 recurrent cancer somatic mutations, and compared them against nine types of regulatory regions. Mendelian disease variants and recurrent cancer somatic mutations are 22- and 10-fold significantly enriched in promoter regions with $\mathrm{q}<0.001$ respectively, compared to allele-frequency-matched genomic background. Different from these two categories, cancer predisposing germline variants are 27-fold enriched in histone modification regions $(\mathrm{q}<0.001)$, 10 -fold enriched in chromatin physical interaction regions $(\mathrm{q}<0.001)$, and 6 -fold enriched in transcription promoter $(\mathrm{q}<0.001)$. However, we observed a dramatic enrichment drop for noncoding cancer predisposing germline variants, with only 3 -fold and 2-fold enrichment in chromatin physical interaction regions and transcription promoter regions with $\mathrm{q}<0.001$, respectively. Furthermore, Mendelian disease variants and recurrent cancer somatic mutations share very similar distributions across types of functional impacts, suggesting the discovery of Mendelian disease variants might be broad enough to cover major pathways.

We also found that nine types of regulatory regions are located within over $50 \%$ of coding exon regions, suggesting the regulatory role of coding regions during gene expression. Transcription promoters, methylation regions, and transcription insulators have the highest density of disease variants, with 472,239 , and 72 disease variants per one million base pairs, respectively.

We recommend that different types of regulatory regions should be investigated for different categories of diseases, and the disease variants curated in this study provide a valuable resource for researchers to investigate the functional impact of disease variants.

\section{Methods}

This study applied computational analytical methods to explore the pathogenic mechanism of disease-associated variants in different disease categories primarily at the regulatory level. 


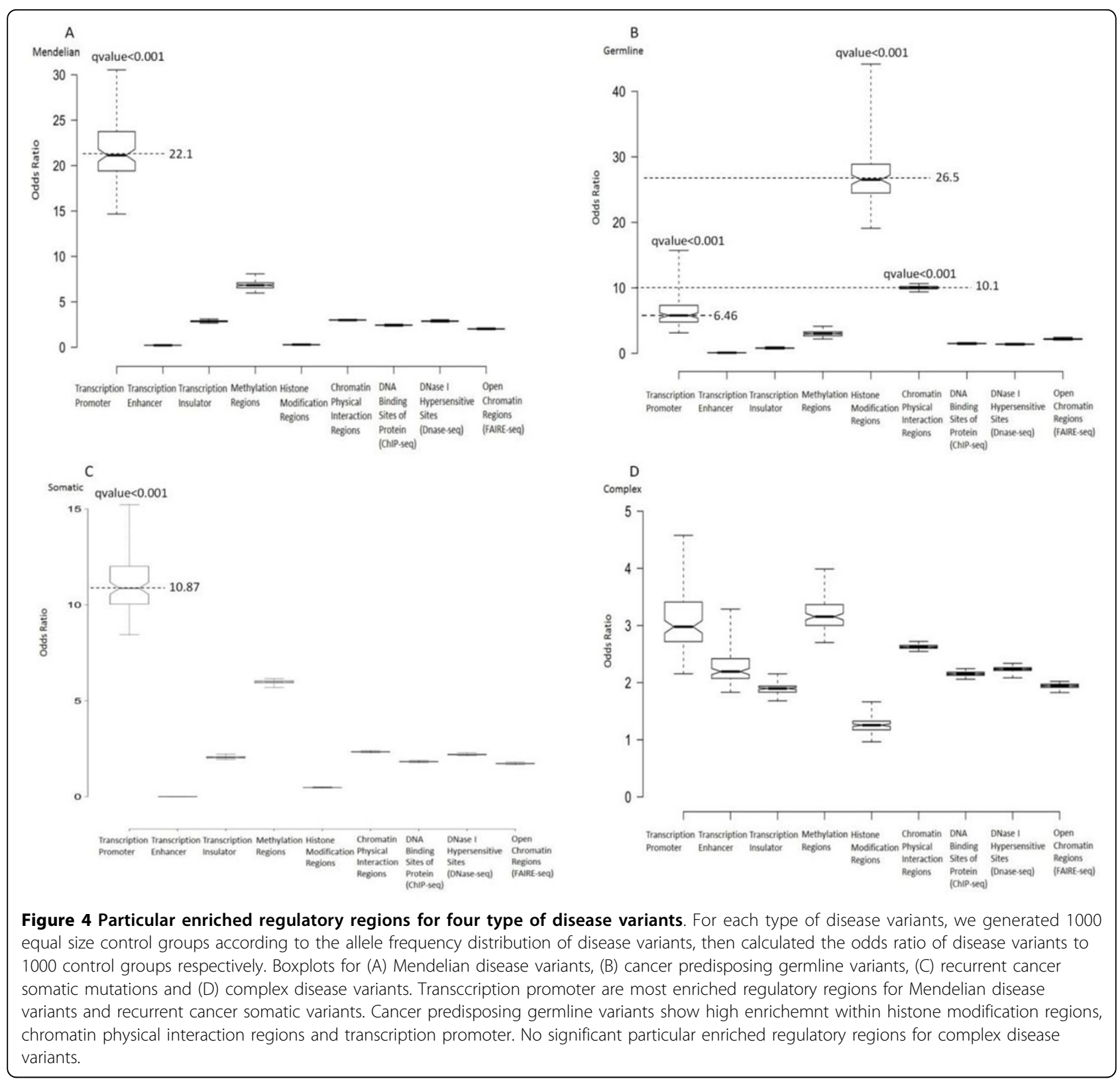

\section{Enrichment analysis}

We compiled all disease-associated variants from multiple data sources. We subtracted disease-associated variants from all SNPs of dbSNP database and considered the remaining SNPs the genome variant background or control group. We then calculated the odds ratio of disease variants to human genome variant background within various regulatory elements. One thousand equal sized specific control groups were generated for each type of disease variants as a further validation experiment. Here we took Mendelian disease variants within promoter regions, for example, to detail how we calculated odds ratio. The Mendelian disease variants were collected from OMIM and ClinVar, and the promoter elements from the FANTOM5 project. The $2 \times 2$ contingency table (Table 5) shows the number of variants that locate within or outside promoter regions for Mendelian variants or control group SNPs. As such, the relative enrichment of Mendelian disease variants to the control group was measured by the resulting odds ratio, which is calculated by the following formula:

$$
O R=\frac{D_{M W} / D_{M O}}{D_{C W} / D_{C O}}
$$

We then calculate the natural logarithm of the odds ratio and the corresponding standard error. The standard 
Table $5.2 \times 2$ contingency table containing the number of Mendelian disease variants and control group SNPs located within or outside promoters for odds ratio calculation

\begin{tabular}{lll}
\hline & Within promoter & Outside promoter \\
\hline Mendelian disease & $D M W$ & $D M O$ \\
Control group & $D C W$ & $D C O$ \\
\hline
\end{tabular}

error for the log odds ratio is calculated by the following formula:

$$
S E=\sqrt{\frac{1}{D_{M W}}+\frac{1}{D_{M O}}+\frac{1}{D_{C W}}+\frac{1}{D_{C O}}}
$$

Lastly, Pearson chi-squared test was performed on the $2 \times 2$ contingency table using a perl module Statistics:: ChisqIndep from CPAN.

\section{Additional material}

Additional File 1: Disease-associated variants for Mendelian diseases and complex diseases, and recurrent cancer somatic mutations. Cancer predisposing germline variants can be downloaded from HGMD Professional.

\section{Competing interests}

The author declares that there is no conflict of interests in relation to this article.

\section{Authors' contributions}

Rong Chen, Meng Ma, Ying Ru designed the study and prepared the manuscript. Jörg Hakenberg and Wei-Yi Cheng collected part of disease variants data and regulatory elements data. Meng Ma did the computational analysis. Ying Ru, Ling-Shiang Chuang, Nai-Yun Hsu and Li-Song Shi interpreted the analysis results. Andrew Uzilov, Wei Ding and Benjamin S. Glicksberg provided many valuable advices.

\section{Acknowledgements}

This study and publication was supported by National Natural and Scientific Funding $(61300057,81000321)$, Anhui Province Natural and Scientific Funding (1208085QF120), and the 48th Scientific Research Staring Foundation for the Returned Overseas Chinese Scholars, Ministry of Education of China (1685). This article has been published as part of BMC Genomics Volume 16 Supplement 8, 2015: Varl-SIG 2014: Identification and annotation of genetic variants in the context of structure, function and disease. The full contents of the supplement are available online at http://www.biomedcentral.com/ bmcgenomics/supplements/16/s8.

\section{Authors' details}

'Department of Genetics and Genomic Sciences, Icahn School of Medicine at Mount Sinai, New York, NY, 10029, USA. ${ }^{2}$ Department of Neuroscience, Icahn School of Medicine at Mount Sinai, New York, NY, 10029, USA. ${ }^{3}$ Department of Endocrinology, Anhui Provincial Hospital, Hefei, Anhui, 230001, China. ${ }^{4}$ School of Computer Science and Technology, Anhui University, Hefei, Anhui, 230039, China.

Published: 18 June 2015

\section{References}

1. Sherry ST, Ward M-H, Kholodov M, Baker J, Phan L, Smigielski EM, Sirotkin K: dbSNP: the NCBI database of genetic variation. Nucleic Acids Research 2001, 29(1):308-311.
2. Elgar $G$, Vavouri $T$ : Tuning in to the signals: noncoding sequence conservation in vertebrate genomes. Trends in Genetics 2008, 24(7):344-352.

3. Consortium EP: An integrated encyclopedia of DNA elements in the human genome. Nature 2012, 489(7414):57-74.

4. Ward LD, Kellis M: Interpreting noncoding genetic variation in complex traits and human disease. Nature Biotechnology 2012, 30(11):1095-1106.

5. Li MJ, Yan B, Sham PC, Wang J: Exploring the function of genetic variants in the non-coding genomic regions: approaches for identifying human regulatory variants affecting gene expression. Briefings in Bioinformatics 2014, pii: bbu018.

6. Stenson PD, Mort M, Ball EV, Howells K, Phillips AD, Thomas N, Cooper DN: The human gene mutation database: 2008 update. Genome Med 2009, 1(1):13.

7. Ponomarenko JV, Merkulova TI, Vasiliev GV, Levashova ZB, Orlova GV, Lavryushev SV, Fokin ON, Ponomarenko MP, Frolov AS, Sarai A: rSNP_Guide, a database system for analysis of transcription factor binding to target sequences: application to SNPs and site-directed mutations. Nucleic Acids Research 2001, 29(1):312-316.

8. Wray GA: The evolutionary significance of cis-regulatory mutations. Nature Reviews Genetics 2007, 8(3):206-216.

9. Cooper DN, Chen JM, Ball EV, Howells K, Mort M, Phillips AD, Chuzhanova N, Krawczak M, Kehrer-Sawatzki H, Stenson PD: Genes, mutations, and human inherited disease at the dawn of the age of personalized genomics. Human Mutation 2010, 31(6):631-655.

10. Welter D, MacArthur J, Morales J, Burdett T, Hall P, Junkins H, Klemm A, Flicek P, Manolio T, Hindorff L: The NHGRI GWAS Catalog, a curated resource of SNP-trait associations. Nucleic Acids Research 2014, 42(D1): D1001-D1006.

11. Gräff J, Tsai L-H: Histone acetylation: molecular mnemonics on the chromatin. Nature Reviews Neuroscience 2013, 14(2):97-111.

12. Haberland M, Montgomery RL, Olson EN: The many roles of histone deacetylases in development and physiology: implications for disease and therapy. Nature Reviews Genetics 2009, 10(1):32-42.

13. FANTOM Consortium and the RIKEN PMI and CLST (DGT), Forrest AR, Kawaji H, Rehli M, Baillie JK, de Hoon MJ, et al: A promoter-level mammalian expression atlas. Nature 2014, 507(7493):462-470.

14. Andersson R, Gebhard C, Miguel-Escalada I, Hoof I, Bornholdt J, Boyd M, Chen Y, Zhao X, Schmidl C, Suzuki T: An atlas of active enhancers across human cell types and tissues. Nature 2014, 507(7493):455-461.

15. Landt SG, Marinov GK, Kundaje A, Kheradpour P, Pauli F, Batzoglou S, Bernstein BE, Bickel P, Brown JB, Cayting P: ChIP-seq guidelines and practices of the ENCODE and modENCODE consortia. Genome Research 2012, 22(9):1813-1831.

16. Song L, Crawford GE: DNase-seq: a high-resolution technique for mapping active gene regulatory elements across the genome from mammalian cells. Cold Spring Harbor Protocols 2010, 2010(2):pdb.prot5384.

17. Fullwood MJ, Liu MH, Pan YF, Liu J, Xu H, Mohamed YB, et al: An oestrogen-receptor-\&agr;-bound human chromatin interactome. Nature 2009, 462(7269):58-64.

18. Kodzius R, Kojima M, Nishiyori H, Nakamura M, Fukuda S, Tagami M, et al: CAGE: cap analysis of gene expression. Nature Methods 2006, 3(3):211-222.

19. Valen E, Pascarella G, Chalk A, Maeda N, Kojima M, Kawazu C, et al: Genome-wide detection and analysis of hippocampus core promoters using DeepCAGE. Genome Research 2009, 19(2):255-265.

20. Salimullah M, Mizuho S, Plessy C, Carninci P: NanoCAGE: a high-resolution technique to discover and interrogate cell transcriptomes. Cold Spring Harbor Protocols 2011, 2011(1):pdb.prot5559.

21. Kanamori-Katayama M, Itoh M, Kawaji H, Lassmann T, Katayama S, Kojima $\mathrm{M}$, et al: Unamplified cap analysis of gene expression on a singlemolecule sequencer. Genome Research 2011, 21(7):1150-1159.

22. Kellis M, Wold B, Snyder MP, Bernstein BE, Kundaje A, Marinov GK, et al: Defining functional DNA elements in the human genome. Proceedings of the National Academy of Sciences 2014, 111(17):6131-6138.

23. Maurano MT, Humbert R, Rynes E, Thurman RE, Haugen E, Wang $H$, et al: Systematic localization of common disease-associated variation in regulatory DNA. Science 2012, 337(6099):1190-1195.

24. Ernst J, Kheradpour P, Mikkelsen TS, Shoresh N, Ward LD, Epstein CB, et al: Mapping and analysis of chromatin state dynamics in nine human cell types. Nature 2011, 473(7345):43-49. 
25. Ernst J, Kellis M: Discovery and characterization of chromatin states for systematic annotation of the human genome. Nature Biotechnology 2010, 28(8):817-825

26. Schaub MA, Boyle AP, Kundaje A, Batzoglou S, Snyder M: Linking disease associations with regulatory information in the human genome. Genome Research 2012, 22(9):1748-1759.

27. Bryzgalov LO, Antontseva EV, Matveeva MY, Shilov AG, Kashina EV Mordvinov VA, Merkulova TI: Detection of Regulatory SNPs in Human Genome Using ChIP-seq ENCODE Data. PLoS one 2013, 8(10):e78833.

28. Karczewski KJ, Dudley JT, Kukurba KR, Chen R, Butte AJ, Montgomery SB, Snyder M: Systematic functional regulatory assessment of diseaseassociated variants. Proceedings of the National Academy of Sciences 2013, 110(23):9607-9612.

29. Farh KK-H, Marson A, Zhu J, Kleinewietfeld M, Housley WJ, Beik S, et al: Genetic and epigenetic fine mapping of causal autoimmune disease variants. Nature 2014, 518:337-343.

30. Ward LD, Kellis M: Interpreting non-coding variation in complex disease genetics. Nature Biotechnology 2012, 30(11):1095-1106.

31. ONline Mendelian Inheritance In Man, OMIM. McKusick-Nathans Institute of Genetic Medicine, Johns Hopkins University (Baltimore, MD); [http://omim. org].

32. Landrum MJ, Lee JM, Riley GR, Jang W, Rubinstein WS, Church DM, Maglott DR: ClinVar: public archive of relationships among sequence variation and human phenotype. Nucleic Acids Research 2013, 42(Database issue):D980-D985.

33. Glicksberg BSLL, Castellanos RZ, Hakenberg J, Cheng W, Khader S, Ma M, et al: An integrative pipeline for multi-modal discovery of disease relationships. Pac Symp Bio 2015, 20:407-418.

34. Forbes SA, Bindal N, Bamford S, Cole C, Kok CY, Beare D, et al: COSMIC: mining complete cancer genomes in the Catalogue of Somatic Mutations in Cancer. Nucleic Acids Research 2010, 39(Database issue) D945-D950.

35. St Laurent G, Shtokalo D, Tackett MR, Yang Z, Eremina T, Wahlestedt C, et al: Intronic RNAs constitute the major fraction of the non-coding RNA in mammalian cells. BMC Genomics 2012, 13(1):504.

36. Relle $M$, Becker $M$, Meyer RG, Stassen M, Schwarting A: Intronic promoters and their noncoding transcripts: A new source of cancer-associated genes. Molecular Carcinogenesis 2014, 53(2):117-124.

37. Ingolia NT, Brar GA, Stern-Ginossar N, Harris MS, Talhouarne GJ, Jackson SE, Wills MR, Weissman JS: Ribosome profiling reveals pervasive translation outside of annotated protein-coding genes. Cell Reports 2014, 8(5):1365-1379.

38. Stergachis AB, Haugen E, Shafer A, Fu W, Vernot B, Reynolds A, et al: Exonic transcription factor binding directs codon choice and affects protein evolution. Science 2013, 342(6164):1367-1372

39. Phillips JE, Corces VG: CTCF: master weaver of the genome. Cell 2009, 137(7):1194-1211.

40. McLaren W, Pritchard B, Rios D, Chen Y, Flicek P, Cunningham F: Deriving the consequences of genomic variants with the Ensembl API and SNP Effect Predictor. Bioinformatics 2010, 26(16):2069-2070.

41. Eilbeck K, Lewis SE, Mungall CJ, Yandell M, Stein L, Durbin R, Ashburner M: The Sequence Ontology: a tool for the unification of genome annotations. Genome Biology 2005, 6(5):R44.

42. Flicek P, Amode MR, Barrell D, Beal K, Billis K, Brent S, et al: Ensembl 2014. Nucleic Acids Research 2013, 42(Database issue):D749-D755.

43. Ritchie GR, Dunham I, Zeggini E, Flicek P: Functional annotation of noncoding sequence variants. Nature Methods 2014, 11(3):294-296.

44. Reva B, Antipin $Y$, Sander C: Predicting the functional impact of protein mutations: application to cancer genomics. Nucleic Acids Research 2011, 39(17):e118.

45. Kircher M, Witten DM, Jain P, O'Roak BJ, Cooper GM, Shendure J: A general framework for estimating the relative pathogenicity of human genetic variants. Nature Genetics 2014, 46(3):310-315.

46. Goode DL, Cooper GM, Schmutz J, Dickson M, Gonzales E, Tsai M, et al: Evolutionary constraint facilitates interpretation of genetic variation in resequenced human genomes. Genome Research 2010, 20(3):301-310.

47. Davydov EV, Goode DL, Sirota M, Cooper GM, Sidow A, Batzoglou S: Identifying a high fraction of the human genome to be under selective constraint using GERP++. PLoS Computational Biology 2010, 6(12): e1001025.
48. Aran D, Sabato $S$, Hellman A: DNA methylation of distal regulatory sites characterizes dysregulation of cancer genes. Genome Biol 2013, 14(3):R21.

49. Portela A, Esteller M: Epigenetic modifications and human disease. Nature Biotechnology 2010, 28(10):1057-1068.

50. Zaidi S, Choi M, Wakimoto H, Ma L, Jiang J, Overton JD, et al: De novo mutations in histone-modifying genes in congenital heart disease. Nature 2013, 498(7453):220-223.

51. Enroth S, Bornelöv S, Wadelius C, Komorowski J: Combinations of histone modifications mark exon inclusion levels. PLoS One 2012, 7(1):e29911.

52. 1000 Genomes Project Consortium, Abecasis, Auton A, Brooks LD, DePristo MA, Durbin RM, et al: An integrated map of genetic variation from 1,092 human genomes. Nature 2012, 491(7422):56-65.

doi:10.1186/1471-2164-16-S8-S3

Cite this article as: Ma et al:: Disease-associated variants in different categories of disease located in distinct regulatory elements. BMC Genomics 2015 16(Suppl 8):S3.

\section{Submit your next manuscript to BioMed Central and take full advantage of:}

- Convenient online submission

- Thorough peer review

- No space constraints or color figure charges

- Immediate publication on acceptance

- Inclusion in PubMed, CAS, Scopus and Google Scholar

- Research which is freely available for redistribution

Submit your manuscript at www.biomedcentral.com/submit
Biomed Central 